\title{
Mobile Writer Verification using a Sequence of Touching Durations in Writing Characters
}

\author{
Takahiro Yoshida, Seiichiro Hangai
}

\begin{abstract}
In on-line signature verification, a set of dynamic raw data such as $x y$ position and pressure of pen, and/or the processed data such as velocity and acceleration data are used as feature parameters. For biometric authentication on mobile devices, however, another feature which is easily extracted from the behavior of finger touching or tipping on screen should be used. For this purpose, we have studied the individuality included in the on-tablet durations in writing characters. In this paper, we describe the characteristics of on-tablet durations in writing characters and their individuality. According to preliminary experiments using name characters written by 11 persons, we can verify the writer without error only by a sequence of on-tablet durations. By using skilled forgery characters, $1.6 \%$ averaged EER (Equal Error Rate) for simulated characters and $3.3 \%$ averaged EER for traced characters are given respectively
\end{abstract}

Keywords-signature, sign, authentiation, verification, biometrics, smartphone, mobile device

\section{Introduction}

As the features of online signature verification, raw time series signature data such as xy position and pressure of pen are used [1]. The processed features used in the verification also include velocity and acceleration of $x y$ position of pen [2], statistics of pen pressure, turning points of xy position and pressure [3] and so on. We have proposed a verification scheme using data of xy position, pressure and inclination of pen and examined its intersession variability [4][5]. In the verification of segmental signature, we have reported that each set of strokes which corresponds to one Japanese character has individuality for verification [6]. In those schemes, high performance tablet, e.g., high sampling frequency such as $100 \mathrm{~Hz}$ or more, high spatial resolution of pen position with 100 pixels $/ \mathrm{mm}$ or more and wide dynamic range of pressure with 1024 levels is used. However, it is difficult to realize the same acquiring condition on mobile devices.

\section{Takahiro Yoshida}

Tokyo University of Science

Japan

Seiichiro Hangai

Tokyo University of Science

Japan
We have studied a possibility of writer verification using the limited DCT coefficients of xy movement in writing signature [7]. This is because the xy movement is one of acquired data available on mobile devices. But, a sequence of on-tablet durations, which is another acquired data have not been used.

In this paper, preliminary experimental results using a sequence of on-tablet durations by 11 writers' handwritten characters are given. After improving the performance of intersession variability, the possibility of writer verification is also discussed based on the EER using genuine and forgery characters.

\section{A Sequence of Touching Durations and Its Variability}

Each stroke in writing characters can be detected by ontablet duration which is measured as a time of non-zero pressure. Fig. 1 shows an example of time series pen pressure $\mathrm{p}(\mathrm{n})$ for four handwritten characters. By determining optimum threshold for pressure data, on-tablet duration $\mathrm{T}(\mathrm{i})$ as a function of stroke position $i$ is measured precisely as shown in Fig. 2. We call this time distribution as a sequence of on-tablet durations.

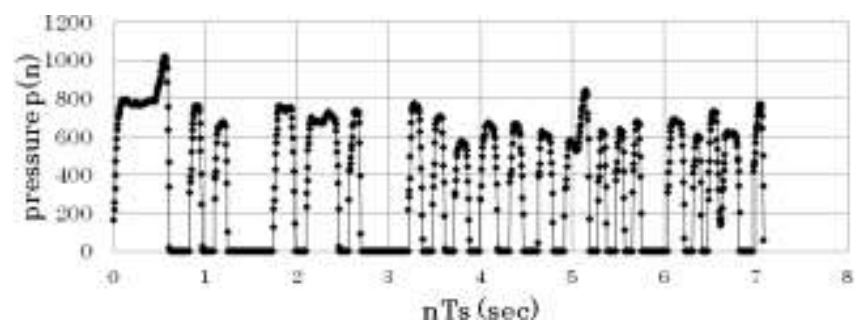

Fig. 1 Example of time series pressure data

From the figure, the number of strokes on different days is equal and the shape of on-tablet duration sequence looks similar. In detail, however, some values at stroke positions 1, 5 , and 13 are different, because writing speeds are affected by writer's conditions and writing environment. This intersession variability is common characteristics to be solved in behavioral biometrics.

For visualizing the similarity, we use the absolute difference distribution $|T(i)-T(j)|$ with respect to all $i$ and $j$ as shown in Fig.3. There is a shallow ditch along $i=j$ and symmetrical pattern with the ditch can be found. This means that stroke positions of two sequences of on-tablet durations are 
Proc. of the Second Intl. Conf. on Advances in Information Processing and Communication Technology - IPCT 2015 Copyright $(\odot$ Institute of Research Engineers and Doctors, USA .All rights reserved.

ISBN: 978-1-63248-044-6 doi: 10.15224/ 978-1-63248-044-6-17

corresponded with each other. As an objective measure of mismatch of two sequences with whole strokes, we use the $\mathrm{SAD}$ (sum of absolute difference) as follows,

$$
S A D=\sum_{k=1}^{K}\left|T(k)-T_{t}(k)\right|
$$

where, $\mathrm{T}_{\mathrm{t}}(\mathrm{k})$ is the template sequence made from several sequence of on-tablet durations, $\mathrm{K}$ is number of strokes of the template and $\mathrm{T}(\mathrm{k})$ is a sequence of on-tablet durations under verification.
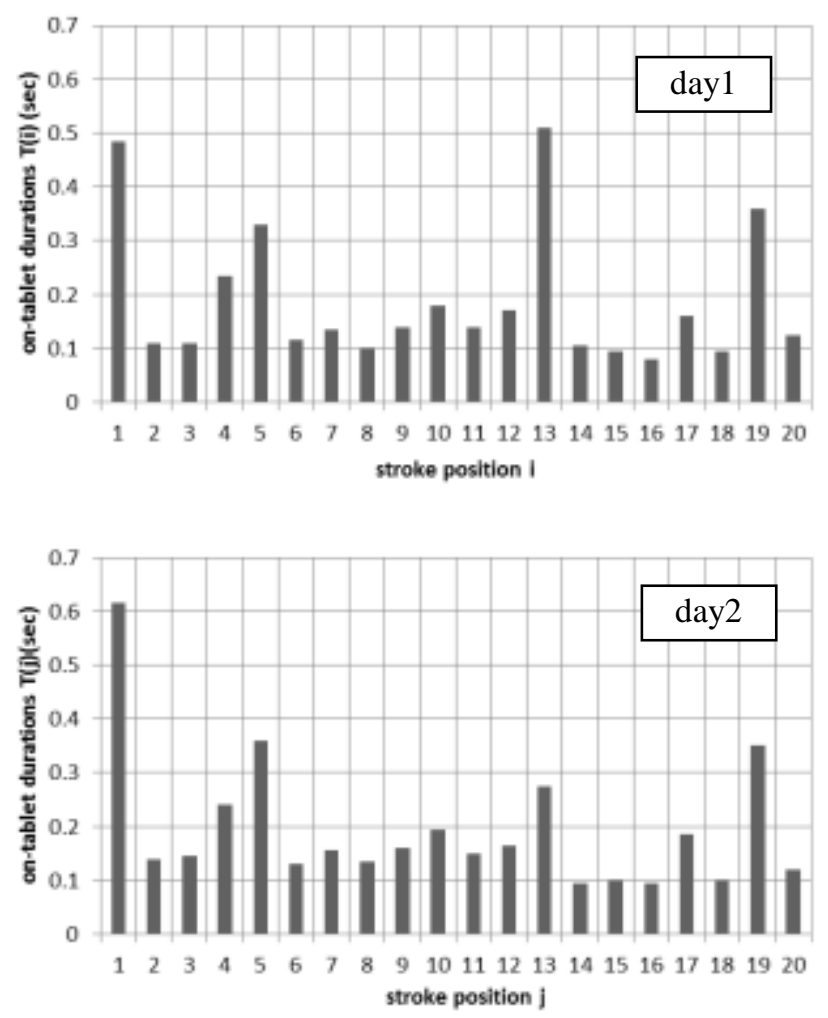

Fig. 2 A sequence of on-tablet durations on different days

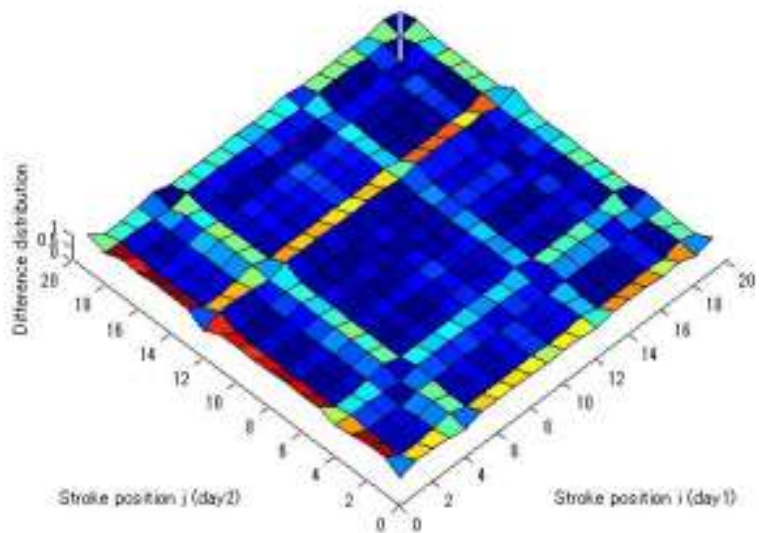

Fig. 3 Absolute difference distribution $|\mathrm{T}(\mathrm{i})-\mathrm{T}(\mathrm{j})|$
In writing sloppy, we often connect adjacent stroke for shortening the writing time. This leads to another variability which must be taken care of. Fig. 4 is another example of two sequences with different number of strokes by the same writer. Fig.5 shows the absolute difference distribution of them. In stroke positions greater than 22, there is little corresponding stroke. This increases the SAD and makes it impossible to accept the sloppy genuine characters. In other words, the difference of number of strokes degrades FRR (False Rejection Rate) characteristics.


Fig. 4 Change of on-tablet durations by emotion

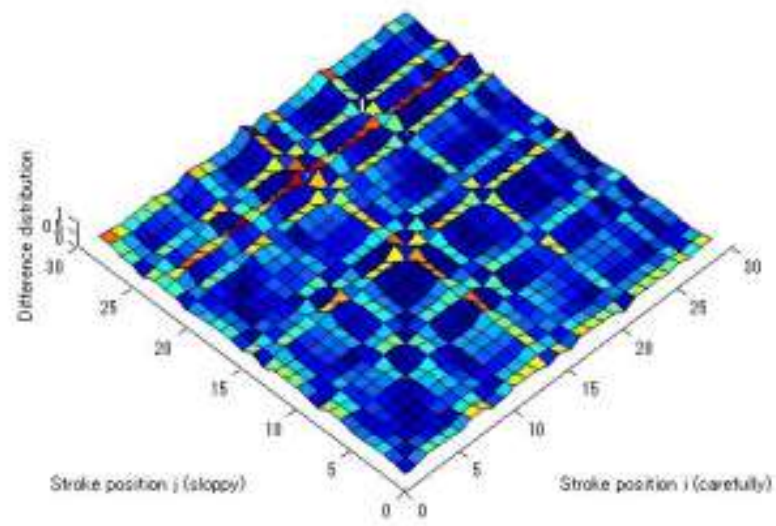

Fig.5 Absolute difference distribution of Fig.4 
Proc. of the Second Intl. Conf. on Advances in Information Processing and Communication Technology - IPCT 2015

Copyright $\odot$ Institute of Research Engineers and Doctors, USA .All rights reserved.

ISBN: 978-1-63248-044-6 doi: 10.15224/ 978-1-63248-044-6-17

\section{DP Matching and Experimental Results}

The robustness against the intersession variability of ontablet durations and number of strokes is a key to realize the reliable authentication system as described in previous section. The FRR is improved by changing the measure of the duration difference from absolute value to relative value. However, the absolute difference of writing time is important to distinguish genuine characters from forgery characters. Therefore, some process such as normalization or rationalization of adjacent duration sometimes degrades the FAR characteristics drastically. By this reason, we use the SAD given by Eq.(1) as the measure of duration difference. Regarding the difference of number of strokes, we use the DP matching which is often used for matching non-linear time series data [8] with narrowing matching window. This window size is determined by the change of the number of strokes by 11 writers.

Fig. 6 shows the flow of verification process using the DP matching algorithm.

In the experiments, each writer must write his/her own name with printed characters by 10 times. In addition, the writer is requested to simulate and trace other names.

Four sequences of on-tablet durations are used in the template creation part. After DP matching, on-tablet durations of corresponding strokes are averaged to make the template. In verification part, only the DP matching is applied before calculating SAD. The evaluation of the performance, i.e., FRR and FAR(False Acceptance Rate) is done by changing SAD threshold.

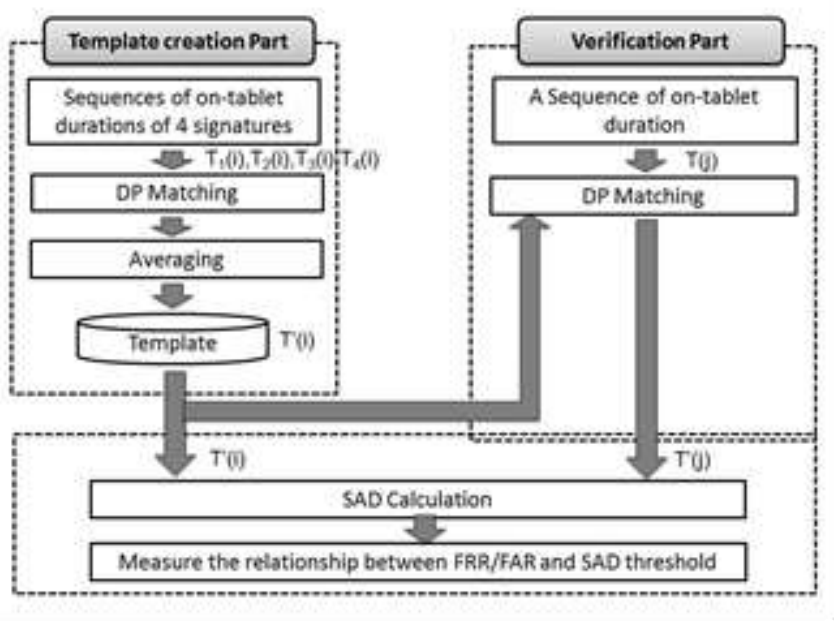

Fig. 6 Writer verification process using on-tablet duration

\section{A. Genuine writer verification}

Fig. 7 shows an example of the relationship between FRR/FAR and the threshold value of SAD without DP matching. Due to intersession variability, the FRR for this writer (TT) is high when the SAD threshold is less than 2 seconds. The EER is $12.9 \%$.

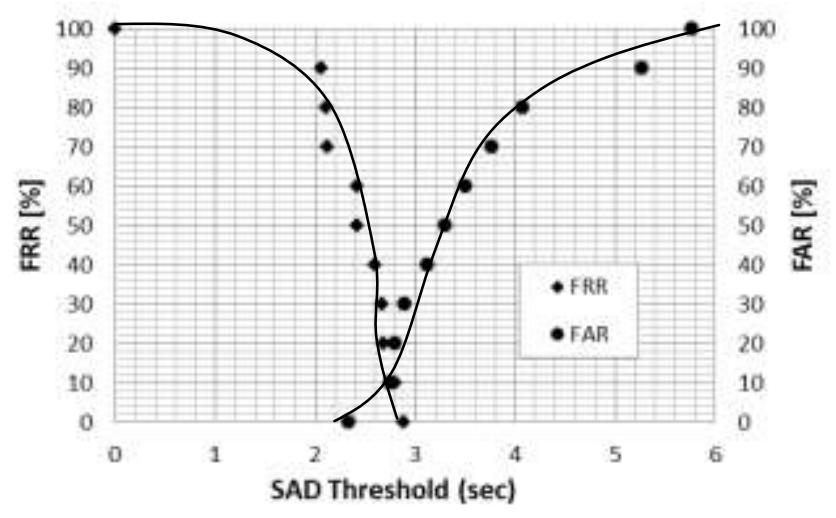

Fig. 7 Relationship between FRR/FAR and SAD threshold without DP matching (writer: TT)

Fig. 8 shows an example of the relationship between FRR/FAR and the SAD threshold of matching error after DP matching with the window of 2 strokes. In comparison with Fig.8, the FRR curve moves to left and no EER for this writer is realized.

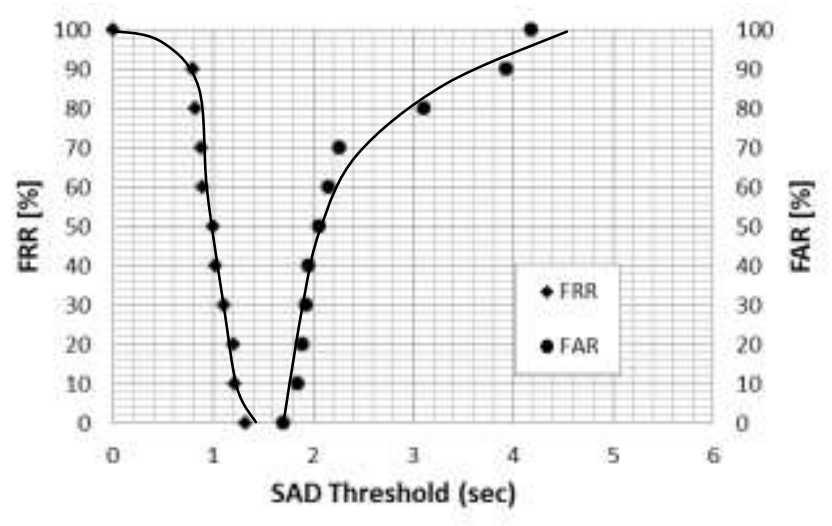

Fig. 8 Relationship between FRR/FAR and SAD threshold after DP matching (writer: TT)

From the result of comparing EERs of genuine characters by 11 writers, the variability of number of strokes affects FRR characteristics and leads to the degradation of EER in case of no DP matching. The averaged EER is $2.8 \%$ in that case. After DP matching, the averaged EER becomes zero and is completely improved.

\section{B. Robustness against forgeries}

There are two types of skilled forgeries. One is simulated forgery, and the other is traced forgery. The former is made by simulating the characters with looking genuine characters. The latter is made by tracing the genuine characters written on a paper. In the experiment, a sequence of on-tablet duration is extracted and DP matched to the template. After calculating 
Proc. of the Second Intl. Conf. on Advances in Information Processing and Communication Technology - IPCT 2015 Copyright $\odot$ Institute of Research Engineers and Doctors, USA .All rights reserved.

ISBN: 978-1-63248-044-6 doi: 10.15224/ 978-1-63248-044-6-17

SAD, the relationship between FAR and SAD threshold is obtained.

Fig. 9 shows an example of the on-tablet durations of simulated forgery and traced forgery in comparison with those of template. From the figure, on-tablet durations in both forgeries are greater than those of genuine characters. This is because a writer needs enough time to mimic another person's characters carefully. Therefore, not only the distribution but also the value itself of on-tablet durations is important feature to distinguish the genuine and the forgery.

Table 1 summarizes the minimum SAD for zero FRR in six handwritten names and the EER using genuine and forgery characters by 11 writers. 8 writers are successfully verified under the conditions of simulated forgery, and 5 writers of them are verified under the condition of traced forgery. The on-tablet sequence of writer KM shows the worst performance in both conditions, because the number of strokes and the writing speed of each stroke change by sessions and FRR characteristics degrade.

Fig. 10 shows the FRR/FAR characteristics of writer KM and KK. From the figure, it is found that the FRR curve of writer KM is staying toward right even if the DP matching is applied. This is the reason why the EER of writer KM degrades.

The averaged EERs of simulated and traced forgery are $1.55 \%$ and $3.26 \%$ respectively.
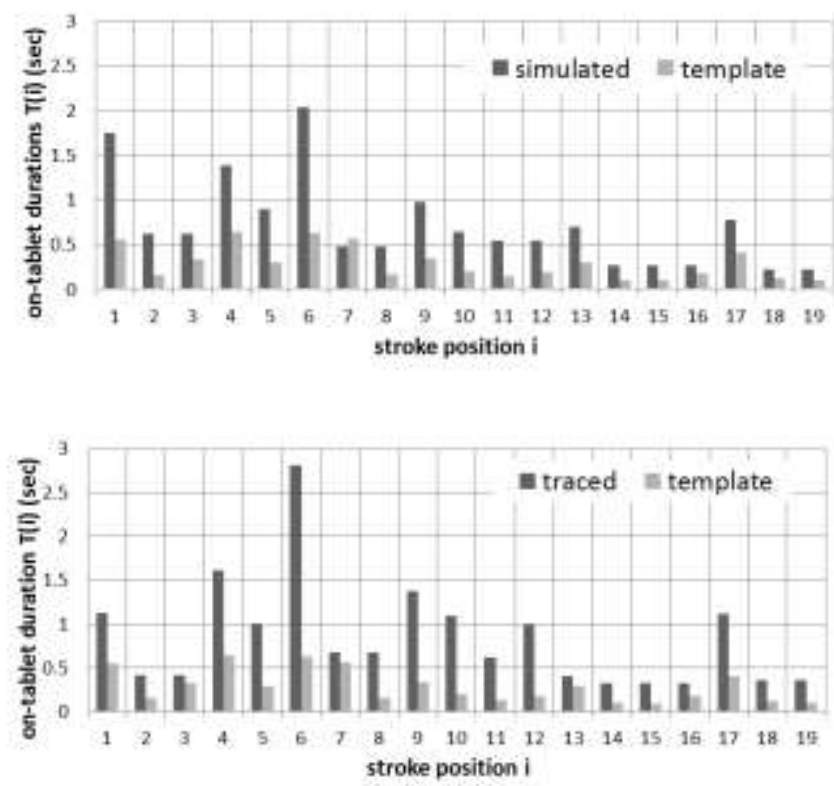

Fig. 9 Difference of on-tablet durations by forgeries
Table 1. EER using genuine and forgery characters

\begin{tabular}{|c|c|c|c|}
\hline Writer & $\begin{array}{c}\text { Min. } \\
\text { SAD for } \\
\text { zero FRR }\end{array}$ & $\begin{array}{c}\text { Forgery } \\
\text { (simulate } \\
\text { d) }\end{array}$ & $\begin{array}{c}\text { Forgery } \\
\text { (traced) }\end{array}$ \\
\hline AT & 0.00 & 0.00 & 0.00 \\
\hline FT & 0.00 & 0.00 & 0.00 \\
\hline IY & 0.00 & 0.00 & 0.00 \\
\hline CS & 0.00 & $2.90 \%$ & $1.66 \%$ \\
\hline KF & 0.00 & 0.00 & 0.00 \\
\hline KM & 2.3 & $7.82 \%$ & $12.55 \%$ \\
\hline KK & 1.1 & 0.00 & $11.27 \%$ \\
\hline TT & 1.35 & 0.00 & 0.00 \\
\hline TYu & 0.00 & 0.00 & $4.31 \%$ \\
\hline TYo & 0.00 & $6.28 \%$ & $5.66 \%$ \\
\hline TA & 0.00 & 0.00 & $0.42 \%$ \\
\hline Average & 0.00 & $1.55 \%$ & $3.26 \%$ \\
\hline
\end{tabular}
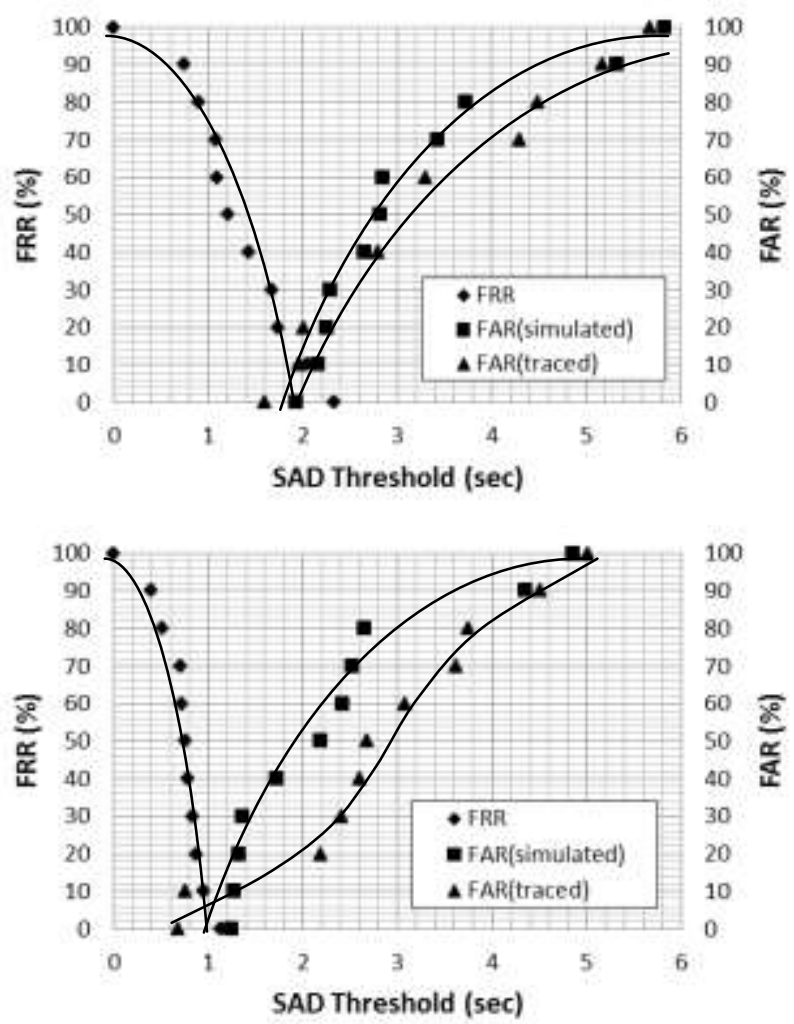

Fig.10 Relationship between FRR/FAR and SAD threshold for writers KM(upper) and KK(lower) 


\section{Conclusion}

In this paper, on-tablet durations as a function of stroke position in writing characters are applied to writer verification. For suppressing the change of number of strokes, DP matching which absorbs the inter session variability of on-tablet information is used.

From the experimental results using characters written by 11 writers, no EER for genuine ones, 1.6\% averaged EER for simulated forgeries, and $3.3 \%$ averaged EER for traced forgeries are obtained respectively. This means that the sequence of on-tablet durations in writing characters could be one of biometric feature for verification on mobile devices. Investigations of feature combination with xy movement using real mobile devices are future subjects.

\section{Acknowledgment}

The authors wish to thank our master course student Ms. Asami Hori for her helpful work regarding experiments.

\section{References}

[1] T.Ohishi, Y.Komiya, T.Matsumoto, 'On-line Signature Verification using Pen-Position, Pen-Pressure and Pen-Inclination Trajectories', ICPR2000, pp547-550, 2000.

[2] A.Kholmatov, B.Yanikoglu, 'Identity Authentication using improved online signature verification method', Pattern Recognition Letter, pp2400-2408, 2005

[3] O.Miguel-Hurtado, L.Mengibar-Pozo, I.Tomeo-Reyes, J.Liu-Jimenez, 'Analysis on compact data formats for the performance of Handwritten Signature Biometrics', Proc. of ICCST, 2009.

[4] S.Hangai, S.Yamanaka, T.Hamamoto, 'On-Line Signature Verification Based On Altitude and Direction of Pen Movement', IEEE ICME2000, Aug. 2000

[5] S.Yamanaka, M.Kawamoto, T.Hamamoto, S.Hangai, 'Signature Verification Adapting to Intersession Variability', IEEE ICME2001, Aug. 2001.

[6] S.Hangai, T.Sano, T.Yoshida, 'A comparison of three kinds of DP matching schemes in verifying segmental signatures', BioID2009, 2009.

[7] T. Yoshida, A. Hori, S. Hangai,' A Study on DCT Coefficients of Time Series Pen Position Data for Printed Signature Verification', ISPA2013, 2013

[8] Hiroaki Sakoe,'Two-Level DP-Matching -A Dynamic ProgrammingBased Pattern Matching Algorithm for Connected Word Recognition', IEEE Trans. of ASSP, ASSP-27, No.6, pp588-595, 1979.

About Author (s):



Takahiro Yoshida

Associate Professor, Tokyo University of Science

His interests include Speech recognition, finger minutiae extraction, Signature verification, etc. He is a member of IEEE

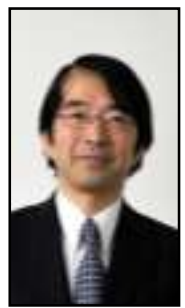

\section{Seiichiro Hangai}

Professor, Tokyo University of Science His interests include Picture quality, Biometrics and Signature verification. $\mathrm{He}$ is a member of IEEE 